\title{
Relação do controle de tronco com a funcionalidade em pacientes hemiparéticos após AVC
}

\author{
Relationship of trunk control with functionality in \\ hemiparetic patients after stroke
}

\section{Relación del control del tronco con la funcionalidad en pacientes hemiparéticos después de un accidente cerebrovascular}

\author{
Paôla Cabral do Prado ${ }^{1}$, Simone Rosa Poletto ${ }^{2}$
}

\begin{abstract}
1.Fisioterapeuta Residente no Programa de Residência Multiprofissional em Saúde do Adulto e Idoso. Universidade Luterana do Brasil - ULBRA. Canoas- RS, Brasil.

2.Fisioterapeuta, Mestre, Docente do Curso de Fisioterapia. Universidade Luterana do Brasil - ULBRA. Canoas- RS, Brasil.
\end{abstract}

\begin{abstract}
Resumo
Objetivo. Avaliar o controle de tronco e funcionalidade em pacientes hemiparéticos pós-AVC através da aplicação das escalas Escala de Deficiência de Tronco (EDT), Escala de Controle de Tronco (ECT), Escala de Avaliação da Simetria e Transferência de Peso (ASTP), Escala de Rankin Modificada (ERm) e Índice de Barthel (IB). Método. Pesquisa quantitativa do tipo transversal, com amostra de 9 indivíduos, atendidos pela fisioterapia na enfermaria do Hospital Municipal de Canoas/RS, de agosto à outubro de 2020. Os dados foram descritos por média e desvio padrão ou mediana e amplitude de variação. Para comparar medianas, o teste de MannWhitney foi utilizado e para correlação dos dados o teste da correlação de Spearman. Resultado. Quanto ao controle de tronco, as medianas encontradas: EDT 10 (0-23), TCT 87 (24-100) e ASTP 18 (9-27). Quanto à funcionalidade, a mediana da ERm foi 4 (0-6) e a média do IB $65 \pm 27,7$ (0-100). Houve associação inversa entre as escalas de controle de tronco e a ERm (EDT $p=0,019$, TCT $p<0,001$ e ASTP $p<0,001$ ) e uma associação positiva com o IB (EDT $p=0,010$, TCT $p<0,001$ e ASTP $p<0,001)$. Conclusão. A relação do controle de tronco com a funcionalidade demonstrou ter uma forte correlação, onde quanto melhor o controle de tronco, maior a funcionalidade.
\end{abstract}

Unitermos. Acidente Vascular Cerebral; Paresia; Fisioterapia; Funcionalidade; Equilíbrio Postural

\begin{abstract}
Objective. To evaluate trunk control and functionality in post-stroke hemiparetic patients by applying the Trunk Impairment Scale (TIS), Trunk Control Test (TCT), Symmetry and Weight Transfer Scale (SWTS), Modified Rankin Scale (mRS) and Barthel Index (BI). Method. Crosssectional quantitative research, with a sample of 9 individuals, attended by physiotherapy in the ward of the Canoas Municipal Hospital/RS, from August to October 2020. The data were described by mean and standard deviation or median and range of variation. To compare medians, the Mann-Whitney test was used and the Spearman correlation test was used to correlate the data. Results. As for trunk control, the medians found: TIS 10 (0-23), TCT 87 (24-100) and SWTS 18 (9-27). As for functionality, the median of mRS was $4(0-6)$ and the mean of the BI $65 \pm 27.7(0-100)$. There was an inverse association between the trunk control scales and the mRS (TIS $p=0.019$, TCT $p<0.001$ and SWTS $p<0.001)$ and a positive association with the BI (TIS $p=0.010$, TCT $p<0.001$ and SWTS $p<0.001$ ). Conclusions. The relationship between trunk control and functionality has been shown to have a strong correlation, where the better the trunk control, the greater the functionality.
\end{abstract}

Keywords: Stroke; Paresis; Physical Therapy; Functionality; Postural Balance 


\section{Resumen}

Objetivo. Evaluar el control y la funcionalidad del tronco en pacientes hemiparéticos después de un ictus aplicando la Escala de deficiencia del tronco (EDT), la Escala de control del tronco (ECT), la Escala de transferencia de simetría y peso (ASTP), la Escala de Rankin modificada $(E R m)$ y el Índice de Barthel (IB). Método. Investigación cuantitativa transversal, con una muestra de 9 individuos, atendidos por fisioterapia en la sala del Hospital Municipal de Canoas / RS, de agosto a octubre de 2020. Los datos se describieron mediante media y desviación estándar o mediana y rango de variación. Para comparar medianas se utilizó la prueba de Mann-Whitney y la prueba de correlación de Spearman para correlacionar los datos. Resultado. En cuanto al control de troncales, las medianas se encontraron: EDT 10 (0-23), TCT 87 (24-100) y ASTP 18 (9-27). En cuanto a la funcionalidad, la mediana de ERm fue de 4 (0-6) y la media de la IB de $65 \pm 27,7(0-100)$. Hubo una asociación inversa entre las escalas de control del tronco y el ERm (EDT $p=0,019$, TCT $p<0,001$ y ASTP $p<0,001$ ) y una asociación positiva con el BI (EDT $p=0,010$, TCT $p<0,001$ y ASTP $p<0,001$ ). Conclusión. La relación entre el control del maletero y la funcionalidad ha demostrado tener una fuerte correlación, donde cuanto mejor es el control del maletero, mayor es la funcionalidad.

Palabras clave. Accidente cerebrovascular; paresia; fisioterapia; funcionalidade; equilibrio postural

Trabalho realizado na Universidade Luterana do Brasil - ULBRA. Canoas- RS, Brasil.

Endereço para correspondência: Paôla C Prado. R. Doutor Barcelos 40, bloco F, apto 404. Bairro Centro. CEP 92310-200. Canoas-RS, Brasil Telefone: (55) 99986-0883. Email: paolacabral@gmail.com

\section{INTRODUÇÃO}

O Acidente Vascular Cerebral (AVC) está entre as principais causas de incapacitação física ou morte, sendo uma doença de grande impacto sobre a saúde da população mundial. No Brasil, as doenças cerebrovasculares são a segunda causa de mortalidade, além de ser um dos maiores motivos de incapacidade e hospitalização ${ }^{1}$. Nas últimas décadas o número de pessoas com o primeiro AVC aumentou em $68 \%$ assim como a taxa de sobrevivência para $84 \%{ }^{2}$. Em 2030, estima-se que o mundo terá 70 milhões de sobreviventes ao AVC, dos quais $70 \%$ não retornarão ao trabalho e 30\% necessitarão de auxílio para locomoção 3 .

Uma das sequelas mais importantes do AVC é a dificuldade na realização dos movimentos, que está 
relacionada à diminuição da função cognitiva, indicando uma forte influência negativa para a recuperação dos movimentos e sobrevivência dos indivíduos acometidos ${ }^{4}$. Outras consequências podem ocorrer, como: alterações de tônus, reações associadas, perda/alteração das reações de equilíbrio, endireitamento e proteção e perda/alteração de movimento seletivo. A hemiplegia é um sinal clássico e é caracterizada por perda dos movimentos voluntários em um hemicorpo, apresentando alterações motoras e possíveis manifestações nos sistemas somatossensorial e cognitivo. Já na hemiparesia, há perda significativa da atividade seletiva em músculos que controlam o tronco, geralmente os músculos que controlam os movimentos de flexão, rotação e flexão lateral ${ }^{5,6}$.

Além do AVC causar déficits no movimento dos membros, marcha e linguagem, o déficit de controle da mobilidade do tronco é também um problema muito significativo. Todas as atividades funcionais normais dependem do controle de tronco como base para 0 movimento. A função dos músculos do tronco é um fator essencial para o balance, transferências, marcha e diversas funções ${ }^{7}$. Desta forma, o tronco deve proporcionar, ao mesmo tempo, estabilidade e mobilidade para que os indivíduos possam realizar suas atividades cotidianas ${ }^{8}$.

Em pacientes pós AVC, a limitação da mobilidade do tronco ocasiona desequilíbrio corporal, consequentemente uma maior insegurança, levando-o a concentrar sua atenção ao tronco, fixando-o para obter maior estabilidade, 
acarretando, assim, aumento da espasticidade dos músculos e deficiências no controle motor. O paciente tende a manter uma postura assimétrica, com distribuição de peso menor sobre o lado mais afetado, transferindo o peso corporal para o lado não afetado. Isso ocorre estando ele sentado ou em pé, passando da posição sentada para a bipedestação ou na marcha. Essa assimetria, a dificuldade em transferir o peso para o lado mais afetado, a espasticidade e a fraqueza muscular interferem na capacidade de manter o controle postural, impedindo/dificultando a orientação e estabilidade para realizar movimentos funcionais com 0 tronco $e$ membros ${ }^{9}$.

Os pacientes com alterações, sejam elas físicas e/ou cognitivas, necessitam de reabilitação com objetivo de restauração funcional, reintegração familiar, comunitária e social, além da manutenção do nível de recuperação e da qualidade de vida, onde se busca uma reabilitação dinâmica, contínua, progressiva e educativa ${ }^{10}$. A Fisioterapia é amplamente usada no processo de reabilitação dos indivíduos acometidos por AVC, tendo como objetivos melhorar a mobilidade funcional, a força muscular, o equilíbrio e a qualidade de vida dos pacientes ${ }^{11}$.

Assim, o presente estudo tem como objetivo principal avaliar o controle de tronco e a funcionalidade em pacientes hemiparéticos, através da aplicação das escalas Escala de Deficiência de Tronco, Escala de Controle de Tronco, Escala de Avaliação da Simetria e Transferência de Peso, Escala de Rankin Modificada e Índice de Barthel. 


\section{MÉTODO}

\section{Amostra}

Esse estudo tratou-se de uma pesquisa quantitativa do tipo transversal com pacientes internados na enfermaria do Hospital Municipal de Canoas, Rio Grande do Sul (RS) submetidos ao atendimento de fisioterapia, entre os meses de agosto a outubro de 2020. Foram incluídos pacientes de ambos os gêneros, com diagnóstico clínico de AVC, hemiparéticos, independente da classe social, lado afetado, etnia, que assinaram o Termo de Consentimento Livre e Esclarecido. Excluiu-se pacientes que no dia da coleta estavam hipertensos, com confusão mental, desorientação têmporo-espacial, não colaborativos e com idade inferior a 18 anos.

Após análise destes critérios, os participantes foram esclarecidos sobre o objetivo da pesquisa e assinaram o Termo de Consentimento Livre e Esclarecido (TCLE), de acordo com a resolução 466/12 do Conselho Nacional de Saúde, após o projeto ter sido aprovado pelo Comitê de Ética e Pesquisa da Universidade Luterana do Brasil (ULBRA) Canoas-RS sob parecer no 4.006.250 e CAAE 29663220.1 .0000 .5349$.

\section{Procedimento}

Os sujeitos foram selecionados por conveniência, onde após aceite do paciente foi preenchido uma ficha de identificação contendo dados como: idade, sexo, tipo de AVC, lado cerebral do AVC, lado afetado, tempo de 
internação, número de atendimentos da fisioterapia e em seguida foram aplicadas as seguintes escalas:

1. Escala de Deficiências de Tronco (EDT): é composta por 17 itens agrupados em três subescalas: equilíbrio estático, equilíbrio dinâmico e coordenação, na postura sentada. As pontuações máximas das subescalas equilíbrio estático, dinâmico e coordenação são 7, 10 e 6, respectivamente. A pontuação total da EDT varia de 0 (pior função de tronco) a 23 (melhor função de tronco). Se a pontuação do paciente é 0 no primeiro item, a pontuação total da EDT é 0 . Será considerada a maior pontuação. Nenhum treino é permitido. $O$ paciente pode ser corrigido entre as tentativas ${ }^{12}$.

2. Escala de Controle de Tronco (TCT): consiste em um instrumento utilizado para avaliar o prejuízo motor em pacientes pós AVC. Em cada teste a pontuação varia de 0 a 25 , sendo que o 0 corresponde à incapacidade da execução da atividade sem ajuda, o 12 a execução do movimento, mas de forma anormal e o 25 a execução da tarefa normalmente. O TCT examina quatro movimentos axiais: rolando de uma posição supina para o lado mais afetado (T1) e para o lado menos afetado (T2), sentando-se a partir de uma posição deitada (T3), e sentando-se em uma posição equilibrada na borda da cama com os pés fora do chão por 30 segundos (T4). A pontuação TCT é a soma das pontuações obtidas nos quatro testes (variação de 0 a 100). A pontuação do examinador deve incidir unicamente sobre o desempenho durante o teste e não se basear em dados referidos. Se o 
teste é feito em 6 semanas após o acidente vascular cerebral uma pontuação maior ou igual a 50 prevê recuperação da capacidade de caminhar em 18 semanas $^{13}$.

3. Avaliação da Simetria e Transferência de Peso (ASTP): a avaliação consiste em analisar a qualidade e o comportamento motor do tronco nas seguintes posturas: sentada, em bipedestação e durante a marcha. Pontos de corte: 8 (ausência de simetria e transferência de peso), 1013 (capacidade mínima de simetria e transferência de peso), 14-18 (capacidade moderada), 19-24 (capacidade parcialmente boa), 25-26 (capacidade boa), 27 (capacidade íntegra) ${ }^{14}$.

4. Escala de Rankin Modificada: avalia o grau de dependência funcional. Possui graus que variam de 0 a 6 e vão desde nenhum grau de incapacidade até morte. Este instrumento de mensuração da incapacidade tem sido amplamente utilizado na avaliação da recuperação neurológica, onde é baseada na incapacidade global (em particular a incapacidade física) e na necessidade de assistência para realizar atividades básicas de vida diária, com ênfase no comprometimento motor ${ }^{15}$.

5. Índice de Barthel: avalia Atividades Básicas de Vida Diária (ABVD) e mede a independência funcional no cuidado pessoal, mobilidade, locomoção e eliminações fisiológicas. Cada item é pontuado de acordo com o desempenho do paciente em realizar tarefas sendo de forma independente, dependente ou com alguma ajuda. A pontuação geral é formada atribuindo-se pontos em cada categoria. A versão 
utilizada avalia a independência funcional em dez tarefas: alimentação, banho, vestuário, higiene pessoal, eliminações intestinais, eliminações vesicais, uso do vaso sanitário, passagem cadeira-cama, deambulação e escadas ${ }^{16,17}$. Ponto de corte: <20 dependência total, 20-35 dependência grave, 40-55 dependência moderada, $\leq 60$ dependência leve ${ }^{18}$.

\section{Análise Estatística}

As variáveis quantitativas foram descritas por média e desvio padrão ou mediana e amplitude de variação. As variáveis categóricas foram descritas por frequências absolutas e relativas. Para comparar medianas, o teste de Mann-Whitney foi utilizado.

Para avaliar a associação entre os escores de controle de tronco e de funcionalidade, o teste da correlação de Spearman foi aplicado. O nível de significância adotado foi de $5 \%(p<0,05)$ e as análises foram realizadas no programa SPSS versão 21.0 .

\section{RESULTADO}

Foram incluídos 9 indivíduos de agosto de 2020 a

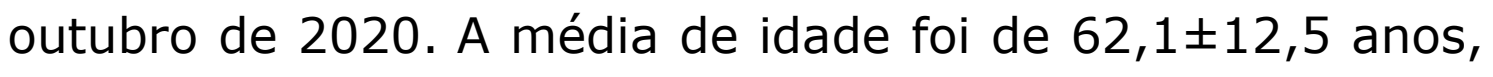
e o principal lado cerebral do AVC foi o direito (100\%). As características clínicas e demográficas dos participantes encontram-se na Tabela 1. 
Tabela 1. Caracterização da amostra.

\begin{tabular}{|c|c|}
\hline Variáveis & $n=9$ \\
\hline Idade (anos) média $\pm \mathrm{DP}$ & $62,1 \pm 12,5$ \\
\hline \multicolumn{2}{|l|}{ Sexo-n (\%) } \\
\hline Masculino & $4(44,4)$ \\
\hline Feminino & $5(55,6)$ \\
\hline Tempo de internação (dias) - média \pm DP & $25,1 \pm 7,8$ \\
\hline Número de atendimentos de fisioterapia - mediana ( $\min -\max )$ & $6(2-14)$ \\
\hline \multicolumn{2}{|l|}{ Tipo de AVC - n (\%) } \\
\hline Isquêmico & $5(55,6)$ \\
\hline Hemorrágico & $4(44,4)$ \\
\hline Lado AVC (Direito) - n (\%) & $9(100)$ \\
\hline \multicolumn{2}{|l|}{ Lado Afetado - n (\%) } \\
\hline Esquerdo & $7(77,8)$ \\
\hline Membros superiores & $1(11,1)$ \\
\hline Membros inferiores & $1(11,1)$ \\
\hline
\end{tabular}

Em relação às variáveis analisadas sobre o controle de tronco, têm-se os seguintes resultados: na EDT, onde avaliam o equilíbrio estático, equilíbrio dinâmico e coordenação, na postura sentada, a mediana encontrada foi 10 (0-23); na TCT a mediana encontrada foi 87 (24-100), onde sabe-se que se 0 teste for feito nas primeiras 6 semanas após o AVC, uma pontuação maior ou igual a 50 prevê recuperação da capacidade de deambular em 18 semanas. Quanto à ASTP, a mediana encontrada foi 18 (927), indicando capacidade moderada. Já em relação à funcionalidade pela Escala Rankin Modificada, a mediana foi de $4(0-6)$, o que indica incapacidade moderada a grave e incapacidade para realizar as atividades e deambular sem ajuda. A média dos valores com relação à funcionalidade mensurada pelo Índice de Barthel foi $65 \pm 27,7$ (0-100), indicando dependência leve (Tabela 2). 
Tabela 2. Escalas de controle do tronco e de funcionalidade.

\begin{tabular}{lc}
\hline Variáveis & $\mathbf{n = 9}$ \\
\hline Escalas de controle de tronco - mediana (min-max) & $10(0-23)$ \\
EDT & $87(24-100)$ \\
TCT & $18(9-27)$ \\
ASTP & $4(0-5)$ \\
Ranking - mediana (min-max) & $65,0 \pm 27,7$ \\
Barthel - média \pm DP & \\
\hline
\end{tabular}

EDT = Escala de Deficiência de Tronco; TCT = Escala de Controle de Tronco; ASTP: Escala de Avaliação da Simetria e Transferência de Peso.

Houve associação inversa significante das escalas de controle de tronco com a de funcionalidade mensurada pela Escala de Rankin modificada, ou seja, quanto maiores os escores de controle de tronco, menores os escores na Rankin, o que indica melhor funcionalidade. Com o Índice de Barthel a associação é positiva, ou seja, quanto maiores os escores de controle de tronco, maiores os escores de Barthel, o que confirma melhor funcionalidade. Assim havendo uma correlação forte entre as escalas TCT, ASTP em relação Barthel e Rankin (Tabela 3).

Tabela 3. Associação entre as escalas de controle de tronco e funcionalidade.

\begin{tabular}{|c|c|c|c|c|}
\hline \multirow{3}{*}{$\begin{array}{l}\text { Escalas de } \\
\text { controle de tronco }\end{array}$} & \multicolumn{4}{|c|}{ Escalas de funcionalidade } \\
\hline & Ranking & & Barthel & \\
\hline & $\begin{array}{l}\text { Coeficiente de } \\
\text { correlação* }\end{array}$ & $\mathbf{P}$ & $\begin{array}{l}\text { Coeficiente de } \\
\text { correlação* }\end{array}$ & $\mathbf{p}$ \\
\hline EDT & $-0,754$ & 0,019 & 0,798 & 0,010 \\
\hline TCT & $-0,922$ & $<0,001$ & 0,948 & $<0,001$ \\
\hline ASTP & $-0,962$ & $<0,001$ & 0,966 & $<0,001$ \\
\hline
\end{tabular}

EDT = Escala de Deficiência de Tronco; TCT = Escala de Controle de Tronco; ASTP: Escala de Avaliação da Simetria e Transferência de Peso. *coeficiente de correlação de Spearman 
Não houve associação significante entre as escalas de controle de tronco e de funcionalidade com a idade, tempo de internação, tipo de AVC e número de atendimentos de fisioterapia.

\section{DISCUSSÃO}

Além do AVC causar déficits no movimento dos membros, marcha e linguagem, o déficit de controle da mobilidade do tronco é um dos comprometimentos mais importantes. Todas as atividades funcionais normais dependem do controle de tronco como base para o movimento. A função dos músculos do tronco é um fator essencial para o equilíbrio, transferências, marcha e diversas funções ${ }^{19}$. Sendo assim, o objetivo do estudo foi avaliar a controle de tronco através da aplicação de três escalas e comparar com a funcionalidade a partir de duas escalas, 0 que nos confirmou que quanto pior for o controle do tronco maior será a perda da funcionalidade. Tal achado gera reflexão importante sobre o impacto do AVC no controle de tronco e funcionalidade, principalmente nas atividades básicas de vida diária (ABVD's).

A aplicação das três escalas, EDT, TCT e ASTP, como ferramenta de avaliação fisioterapêutica, demonstra que durante o tratamento fisioterapêutico se deve estimular mais o equilíbrio, a simetria corporal e a transferência de peso, pois não se teve resultado significativo sobre o controle de tronco destes pacientes. Sendo assim, cada vez mais a busca por métodos avaliativos eficientes e precisos tem sido uma 
constante para planejar e executar uma intervenção mais efetiva, sendo uma forma de quantificar as alterações motoras e suas respectivas evoluções ${ }^{20}$.

Assim como nosso estudo que correlacionou controle de tronco, onde uma das variáveis é o equilíbrio, com a funcionalidade, outro estudo avaliou 24 hemiparéticos, e também correlacionou $\mathrm{o}$ déficit de equilíbrio com 0 comprometimento motor nas atividades funcionais através da Medida de Independência Funcional (MIF), da Escala de Equilíbrio de Berg (EEB), do Índice do Andar Dinâmico (IAD) e do Protocolo de Desempenho Físico da Fugl-Meyer (FM), onde foi encontrada correlação positiva entre o equilíbrio, nível de independência funcional e comprometimento motor da extremidade inferior dos hemiparéticos ${ }^{21}$.

Foi observada uma coerência entre o nosso estudo e outro estudo que comparou simetria corporal e controle de tronco em hemiparéticos pós $\mathrm{AVC}^{22}$. Foram usadas as escalas ASTP e EDT, onde foi observado que a fraqueza, o aumento do tônus nos músculos do tronco, aumento da oscilação postural, instabilidade dinâmica, e dificuldades na transferência de peso afetam negativamente o desempenho nas atividades, assim como o controle de tronco é um dos indicadores mais importantes de recuperação funcional pós $A V C$, tendo uma forte correlação entre o desempenho do tronco e o equilíbrio, a marcha e as habilidades funcionais nas ABVD's.

Com o objetivo de caracterizar pacientes com AVC, correlacionando os achados da EDT com área da lesão, 
idade, quadro clínico e estágio de evolução, outro estudo não encontrou relação entre a pontuação da EDT, o quadro clínico, a evolução e algumas características dos indivíduos ${ }^{23}$, corroborando com os achados do presente estudo. Sendo assim, mudanças em diferentes variáveis não interferiram em sua pontuação, como a idade, o sexo, os tipos de AVC, o quadro clínico, as áreas comprometidas e o estágio em que o paciente se encontrava na avaliação, pois a EDT tem por objetivo apenas avaliar o equilíbrio sentado pós-AVC, o que inclui o equilíbrio estático e dinâmico e a coordenação de tronco durante tal posição. Já em outro estudo foi encontrada alta correlação entre Barthel e a pontuação da EDT somente na fase aguda do $\mathrm{AVC}^{24}$. Com o passar do tempo, a variabilidade na evolução do quadro agudo gerou resultados diversificados.

Com relação ao controle postural, foi investigado os determinantes do desequilíbrio postural em 186 pacientes geriátricos após AVC, e observaram que a influência da força muscular sobre o controle postural é mais presente em pacientes com nível básico de controle de tronco e que a força muscular do lado do corpo mais afetado é um fator determinante do controle postural em pacientes com AVC 25 . Esses problemas podem limitar a taxa de recuperação de deambular e de independência funcional, podendo levar a distúrbios na marcha, equilíbrio e $\mathrm{ABVDs}^{26}$.

O controle de tronco é um importante preditor da capacidade para executar ABVDs, sendo recomendada sua avaliação precoce ${ }^{27}$. Portanto, a avaliação do controle de 
tronco talvez seja o melhor indicativo de ganhos funcionais. O exercício para o tronco aumentou a atividade muscular do tronco e melhorou a capacidade de controle postural durante o movimento funcional em 24 pacientes com AVC, apresentando melhora na funcionalidade destes pacientes ${ }^{28}$.

A presente pesquisa apresentou limitações que impossibilitam a generalização dos resultados. Não foi possível obter uma amostra com mais participantes devido ao número reduzido de internações por AVC, dentro dos critérios de inclusão do estudo, devido à pandemia pelo SarsCov-2, pois o hospital tornou-se referência no tratamento da COVID-19. Outra limitação foi o curto tempo disponibilizado para a coleta dos dados diante da reduzida circulação de pessoas nas áreas de internação hospitalar.

\section{CONCLUSÃO}

A relação do controle de tronco com a funcionalidade demonstrou ter uma forte associação positiva, onde quanto melhor o controle de tronco, maior a funcionalidade. Com relação às ABVD's e o controle de tronco, observou-se, no presente estudo, correlação indireta forte, onde quanto melhor o controle de tronco, mais independente é o paciente nas suas atividades diárias, na amostra estudada.

Mais estudos precisam ser realizados, com amostras maiores e maior tempo de acompanhamento para generalização dos resultados à população. 


\section{REFERÊNCIAS}

1.Araújo JPD, Darcis JVV, Tomas ACV, Mello WAD. Tendência da mortalidade por acidente vascular cerebral no município de Maringá, Paraná entre os anos de 2005 a 2015. Inter J Cardiovas Sci 2018;31:56-62. http://dx.doi.org/10.5935/2359-4802.20170097

2.Santos EB, Rodrigues RA, Marques S, Pontes-Neto OM. Estresse percebido nos idosos sobreviventes do AVC após a alta hospitalar para casa. Rev EsC Enferm USP 2015;49:797-803. http://dx.doi.org/10.1590/S0080-623420150000500013

3.Brasil. Ministério da Saúde. Linha de Cuidados em Acidente Vascular Cerebral ( $A \vee C)$ na rede de Atenção às Urgências e Emergências (endereço na internet). Brasília: Ministério da Saúde; 2012 (acessado em: 10/12/2019). Disponível em: https://bvsms.saude.gov.br/bvs/publicacoes/manual rotinas para at encao avc.pdf

4.Costa FA, Silva DLA, Rocha VM. Estado neurológico e cognição de pacientes pós-acidente vascular cerebral. Rev EsC Enferm USP 2011;45:1083-8.

http://dx.doi.org/10.1590/S0080-

62342011000500008

5.Piassaroli CAP, Almeida GC, Luvizotto JC, Suzan ABBM. Modelos de Reabilitação Fisioterápica em Pacientes Adultos com Sequelas de AVC Isquêmico. Rev Neurocienc 2012;20:128-37. http://dx.doi.org/10.34024/rnc.2012.v20.10341

6. Martin A, Abogunrin S, Kurth H, Dinet J. Epidemiological, humanistic, and economic burden of illness of lower limb spasticity in adults: a systematic review. Neuropsychiatr Dis Treat 2014;10:111-22. http://dx.doi.org/10.2147/ndt.553913

7.Antunes JE, Justo FHO, Justo AFO, Ramos GC, Prudente COM. Influência do controle postural e equilíbrio na marcha de pacientes com sequela de acidente vascular cerebral. Fisiot Saúde Func 2016;5:3041. http://www.repositorio.ufc.br/handle/riufc/19343

8.Van Criekinge T, Saeys W, Hallemans A, Vereeck L, De Hertogh W, Van de Walle $P$, et al. Effectiveness of additional trunk exercises on gait performance: study protocol for a randomized controlled trial. Trials 2017;18:249. http://dx.doi.org/10.1186/s13063-017-1989-1

9.Schuster RC. Correlação entre Disfunções Motoras e Respiratórias no AVC. Rev Neurocienc 2011;19:587-8. http://dx.doi.org/10.34024/rnc.2011.v19.8319

10.Rangel ESS, Belasco AGS, Diccini S. Qualidade de vida de pacientes com acidente vascular cerebral em reabilitação. Acta Paul Enferm 2013;26:205-12.

http://dx.doi.org/10.1590/S0103-

21002013000200016

11.Brito R, Lins LCRF, Almeida CDA, Ramos Neto ES, Araújo DP, Franco CIF. Instrumentos de Avaliação Funcional Específicos Para o Acidente Vascular Cerebral. Rev Neurocienc 2013;21:593-9. http://dx.doi.org/10.34024/rnc.2013.v21.8145

12.Verheyden G, Nieuwboer A, Mertin J, Preger R, Kiekens C, Weerdt WD. The trunk impairment scale: a new tool to measure motor 
impairment of the trunk after stroke. Clin Rehab 2004;18:326-34. http://dx.doi.org/10.1191/0269215504cr733oa

13.Fujiwara T, Liu M, Tsuji T, Sonoda S, Mizuno K, Akaboshi K, et al. Development of a new measure to assess trunk impairment after stroke (Trunk Impairment Scale): its psychometric properties. Am J Phys Med Rehabil 2004;83:681-8.

http://dx.doi.org/10.1097/01.PHM.0000137308.10562.20

14.Chagas EF, Tavares MDCGC. A simetria e transferência de peso do hemiplégico: relação dessa condição com o desempenho de suas atividades funcionais. Fisiot Pesq 2001;8:40-50. http://dx.doi.org/10.1590/fpusp.v8i1.79397

15.Wilson JTL, Hareendran A, Hendry A, Potter J, Bone I, Muir KW. Reliability of the modified Rankin Scale across multiple raters: benefits of a structured interview. Stroke 2005;36:777-81. http://dx.doi.org/10.1161/01.STR.0000157596.13234.95

16. Minosso JSM, Amendola F, Alvarenga MRM, Oliveira MAC. Validação, no Brasil, do Índice de Barthel em idosos atendidos em ambulatórios. Acta Paul Enferm 2010;23:218-23. http://dx.doi.org/10.1590/S010321002010000200011

17.Fluetti MT, Fhon JRS, Oliveira APD, Chiquito LMO, Marques S. Síndrome da fragilidade em idosos institucionalizados. Rev Bras Geriatr Gerontol 2018;21:60-9. http://dx.doi.org/10.1590/198122562018021.170098

18.Vasconcelos TG, Reis LA, Reis LA, Fernandes MH, Alves GS, Sampaio LS, et al. Funcionalidade familiar de idosos dependentes residentes em domicílios. Aval Psicol 2009;8:415-23. http://www.redalyc.org/articulo.oa?id $=335027282013$

19.Trindade APNT, Barboza MA, Oliveira FB, Borges APO. Influência da simetria e transferência de peso nos aspectos motores após Acidente Vascular Cerebral. Rev Neurocienc 2011;19:61-7. http://dx.doi.org/10.34024/rnc.2011.v19.8402

20.Sena CG, Saes MO, Brod M, Pitzer Neto VE. Eficácia do tratamento fisioterápico para o controle de tronco em indivíduos acometidos pelo acidente vascular encefálico. Rev Insp Mov Saúde 2013;5:16-9. https://www.inspirar.com.br/wp-content/uploads/2014/01/eficaciado-tratamento-artigo361.pdf

21.Azevedo ERFBM, Macedo LS, Paraízo MFN, Oberg TD, Lima NMFV, Cacho EWA. Correlação do déficit de equilíbrio, comprometimento motor e independência funcional em indivíduos hemiparéticos crônicos. Acta

Fisiatr 2008;15:225-8. http://www.periodicos.usp.br/actafisiatrica/article/download/102995/ 101280

22.Souza JG, Souza RB, Costa VDSP, Ossada VAY, Tsukamoto HF. Avaliação da Simetria Corporal e Controle de Tronco em Hemiparéticos Pós-Acidente Vascular Encefálico: Uma Série de Casos. Rev Equil Corp Saúde 2017;8:11-6. $\quad$ http://dx.doi.org/10.17921/21769524.2016v8n1p11-16

23. Caires TA, Silva GV, Castro SS, Souza LAPS. Controle de tronco e sua relação com quadro clínico, área comprometida e fase pós-acidente 
vascular encefálico. Fisiot Pesq 2018;25:224-8. http://dx.doi.org/10.1590/1809-2950/17025025022018

24.Kim TJ, Seo KM, Kim D, Kang SH. The relationship between initial trunk performances and functional prognosis in patients with stroke. Ann Rehabil Med 2015;1:66-73. http://dx.doi.org/10.5535/arm.2015.39.1.66

25.Park $\mathrm{YH}$, Lee $\mathrm{C}$, Lee $\mathrm{BH}$. Clinical usefulness of the virtual realitybased postural control training on the gait ability in patients with stroke. J Exerc Rehabil 2013;9:489-94. http://dx.doi.org/10.12965/jer.130066

26. Belchior ACS. Avaliação da funcionalidade de pacientes com sequelas de acidente vascular cerebral por meio da escala MIF. Fisiot Bras 2018;19:208-17. https://doi.org/10.33233/fb.v19i5.2624

27. Hsieh CL, Sheu CF, Hsueh IP, Wang CH. Trunk control as an early predictor of comprehensive activities of daily living function in stroke patients.

Stroke 2002;33:2626-30.

http://dx.doi.org/10.1161/01.STR.0000033930.05931.93

28.Jung KS, Cho HY, In TS. Trunk exercises performed on an unstable surface improve trunk muscle activation, postural control, and gait speed in patients with stroke. J Phys Ther Sci 2016;28:940-4. http://dx.doi.org/10.1589/jpts.28.940 